Institut für Angewandte Pbysik der Universität Regensburg, W.-Germany und Abteilung Exp. Pbysik III, Universität Ulm, W.-Germany

\title{
Phonon mode coupling and Fano-type antiresonance in polyethylene
}

\author{
W. Prettl and W. F. X. Frank
}

With 6 figures

\section{Introduction}

Polyethylene (PE) is a nonpolar partially crystalline polymer. The crystalline regions have lateral dimension of several hundred Angstroms and are embeded in an amorphous polymer matrix. Crystalline polyethylene belongs to the orthorhombic crystal system with point group $D_{2 h}$. In the far infrared spectral region $\mathrm{PE}$ shows a strong absorption line at $73 \mathrm{~cm}^{-1}$ and a very weak band at $109 \mathrm{~cm}^{-1}$, which is observable only at low temperature up to about $120 \mathrm{~K}[1,2]$. Both bands are superimposed on a continuous background absorption. They have been recognized to result from external lattice vibrations of the crystalline portions of the polymer $[3,4]$. The corresponding optical phonons belong to the $B_{1 u}\left(73 \mathrm{~cm}^{-1}\right)$ and $B_{2 u}\left(109 \mathrm{~cm}^{-1}\right)$ representations of $D_{2 h}$, where, as shown in figure 1 , the two sublattices vibrate along the $b$-axis and in $a$-axis direction, respectively. The transition dipole moments of both modes are normal to the elongations of the PE-chains.

A recent detailed investigation of the temperature dependence of the far infrared absorption of PE by Frank et al. has revealed several remarkable features of the structure of the absorption which have not yet been satisfactorily explained [5]. Figure 2 shows the absorption coefficient of $\mathrm{PE}$ as a function of frequency for several temperatures between $4 \mathrm{~K}$ and $414 \mathrm{~K}$, just below the melting temperature $T_{m}=430 \mathrm{~K}$. The weak $B_{2 u}$-band decreases with rising temperature and vanishes at $120 \mathrm{~K}$. The absorption strength of the $B_{1 u}$-band is constant up to $80 \mathrm{~K}$. Above that temperature the absorption decreases gradually

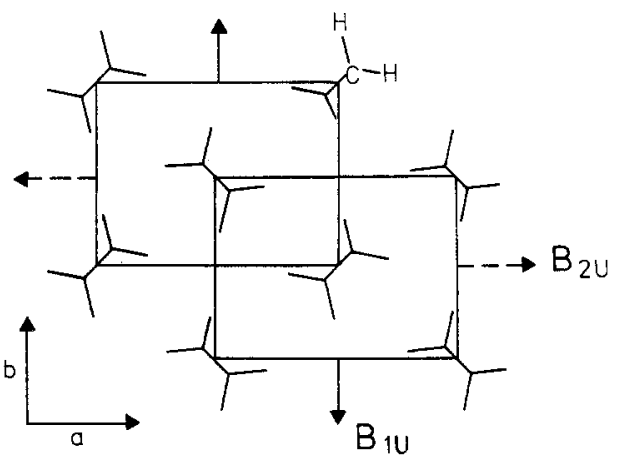

Fig. 1. Cross section of unit cell of PE. The arrows indicate the translational motion of the $B_{1 u}\left(73 \mathrm{~cm}^{-1}\right)$ phonon (full arrow) and the $B_{2 u}\left(109 \mathrm{~cm}^{-1}\right)$ phonon (broken arrow)

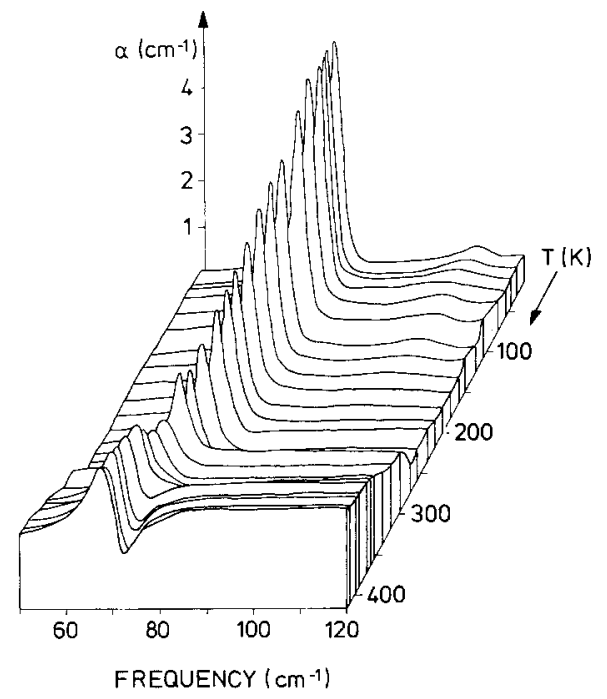

Fig. 2. Far infrared absorption of PE (6011 L) at various temperatures after [5] 
in contrast to the behaviour of the absorption strength of a usual Reststrahlen-phonon. The absorption line is symmetric up to $310 \mathrm{~K}$ as it is expected for a narrow one phonon line. Above $310 \mathrm{~K}$ the line becomes more and more asymmetric and a pronounced antiresonance emerges at the high frequency wing of the line, which becomes deeper with rising temperature. In the following we will give a conceivable explanation for this anomalous behavior of the $B_{1 u}$-line within the framework of a simple phenomenological theory.

\section{Theory}

The strong antiresonance suggests an explanation according to Fano's treatment of interference distortion of atomic transitions [6]. Basically the line shape of a transition between discrete energy levels may be distorted by interference of the states of the discrete spectrum with those of a continuous spectrum. Similar effects have also been found for various interacting excitations in solids $[7,8]$. The measurements shown in figure 2 represent the first observation of a Fano-type antiresonance in a polymer.

For polyethylene we assume that the amplitude $X_{0}$ of the $B_{1 u}$ optical phonon is linearly coupled with the amplitudes $X_{\mu}$ of an infrared active continuum of vibrational modes. This continuum is at least partially responsible for the continuous background absorption. The most general dynamical equation of the coupled vibrational modes driven by an external electric field $E$ has in quasi harmonic approximation the form

$$
\begin{aligned}
& \left(\omega_{0}^{2}-\omega^{2}-i \omega \gamma_{0}\right) X_{0}+\sum_{\mu} L_{0 \mu} X_{\mu}=Z_{0} E \\
& L_{\mu 0} X_{\mu}+\left(\omega_{\mu}^{2}-\omega^{2}-i \varepsilon\right) X_{\mu}=Z_{\mu} E \\
& \mu=1 \ldots N
\end{aligned}
$$

where $Z_{0}$ and $Z_{\mu}$ are suitably normalized effective charges [9] containing local field corrections, and $L_{0 \mu}=L_{\mu 0}$ are phenomenological coupling coefficients being generally complex. The resonance frequency and the damping constant of the uncoupled discrete phonon of amplitude $X_{0}$ are $\omega_{0}$ and $\gamma_{0}$, respectively, $\omega_{\mu}$ is the frequency of a continuum vibration and $\varepsilon$ is infinitesimal as we will not take into account explicitely a finite damping of the continuum modes. Our model defined by equation 1 is an extension of Barker and Hopefield's theory of the infrared dispersion of coupled optical phonons [9]. A mechanical analogue of the coupled system of oscillators may be set up as shown figure 3. Elongation of a continuum vibration excerts a force on the discrete oscillator and the elongation of the discrete mode excerts forces on all continuum modes according to the coupling coefficients $L_{0 \mu}$. The coupling establishes fixed phase relations among the modes. The antiresonance in the absorption spectrum occurs due to destructive interference of the dipole moments of the discrete phonon $Z_{0} X_{0}$ and those of the continuum modes $\sum_{\mu} Z_{\mu} X_{\mu}$.

The electric susceptibility of the system of coupled oscillators is given by

$\chi=\sum_{i, j=0}^{N} Z_{i} G_{i j} Z_{j}+\chi_{\infty}$

where $\chi_{\infty}$ is the (real) susceptibility at optical frequencies and the response matrix $G=G_{i j}$ of the coupled system is defined by

$$
\begin{aligned}
& g_{0}^{-1} L_{01} \ldots L_{0 N} \\
& L_{10} g_{1}^{-1} 0 \ldots 0 \\
& \underline{G}^{-1}= \\
& 0 \\
& L_{N 0} \quad g_{N}^{-1} .
\end{aligned}
$$

Here $g_{0}=\left(\omega_{0}^{2}-\omega^{2}-i \omega \gamma_{0}\right)^{-1}$ and $g_{\mu}=\left(\omega_{\mu}^{2}-\right.$ $\left.\omega^{2}-i \varepsilon\right)^{-1}$ are the classical response functions of the uncoupled discrete phonon and the continuum vibrations, respectively.

The matrix $G$ can easily be determined. We find:

$$
G_{00}=G_{0}
$$

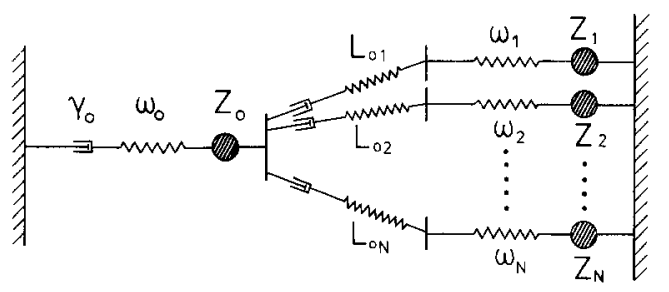

Fig. 3. Mechanical model illustrating the coupled oscillator system defined by equation 1 
$G_{0 \mu}=-L_{0 \mu} g_{\mu} G_{0}$

$G_{\mu \gamma}=g_{\mu} \delta_{\mu \nu}+L_{0 \mu} g_{\mu} L_{0 \gamma} g_{\gamma} G_{0}$

$\mu, \gamma=1 \ldots N$.

In these expressions

$G_{0}=\left(g_{0}^{-1}-\Delta\right)^{-1}=\left(\Omega_{0}^{2}-\omega^{2}-i \omega \Gamma_{0}\right)^{-1}$

with

$\Delta=\Delta^{\prime}+i \Delta^{\prime \prime}=\sum_{\mu=1}^{N} L_{0}^{2} g_{\mu}$,

$\Omega_{0}^{2}=\omega_{0}^{2}-A^{\prime}$,

$\omega \Gamma_{0}=\omega \gamma_{0}+\Delta^{\prime \prime}$ and

$\delta_{\mu \gamma}$ is the Kronecker symbol.

Inserting the response function equation 4 into equation 2 we can write the electric susceptibility as a sum:

$\chi=\chi_{c}+\chi_{d}+\chi_{\infty}$

where

$\chi_{c}=\sum_{\mu=1}^{N} Z_{\mu}^{2} g_{\mu}$

is the contribution of the vibrational continuum and

$\chi_{d}=F_{0}^{2} G_{0}$

with $F_{0}=Z_{0}-\Lambda$ and

$\Lambda=\Lambda^{\prime}+i \Lambda^{\prime \prime}=\sum_{\mu=1}^{N} Z_{\mu} L_{0 \mu} g_{\mu}$

is the susceptibility due to the discrete phonon. Equations 7 and 8 shows that $\chi_{c}$ is unaffected by the interaction whereas the response of the discrete phonon is renormalized. The resonance frequency and the damping constant of the interacting discrete phonon are $\Omega_{0}=$ $\omega_{0}-\Delta^{\prime}$ and $\Gamma_{0}=\gamma_{0}-\Delta^{\prime \prime} / \omega$, respectively, where the "self energy" $\Delta$ depends only on the coupling coefficients $L_{0 \mu}$. Thus the frequency renormalization may occur even if the continuous spectrum is optically inactive as it is usually the case if a well defined excitation interacts with a continuum. The coupling also changes the effective charge of the discrete phonon which becomes $F_{0}=Z_{0}-A$. In contrast to $\Delta$ the coefficient $A$ depends on the coupling coefficients $L_{0 \mu}$ and the charges of the continuum phonons $Z_{\mu}$. Thus the occurrence of $A$ requires the coupling of the discrete phonon with continuous excitations and the infrared activity of the continuum modes. Because $A$ is generally a complex quantity, $F_{0}$ and thus $F_{0}^{2}$ become complex. This is just the reason why the absorption line shape of the discrete phonon deviates from that of a simple damped oscillator and may show an antiresonance. The susceptibility $\chi$ as given by equations 6 to 8 is formally equal to the composite electron-phonon Green's function, which has been derived by Balkanski et al. [8] in discussing distorted Raman-scattering lines shapes in semi-conductors. It is obvious that the physical meanings of the parameters $\Delta$ and $A$ are different.

The optical absorption coefficient is given by

$\alpha(\omega)=\frac{4 \pi \omega}{n c} \operatorname{Im} \chi$

where $n$ is the refractive index and $c$ the velocity of light. According to equation $6 \propto(\omega)$ may be decomposed into:

$\alpha(\omega)=\alpha_{c}(\omega)+\alpha_{d}(\omega)$

with $\alpha_{c}$ the absorption of the continuous background and $\alpha_{d}$ the absorption coefficient of the renormalized discrete phonon. In the limit $\varepsilon \rightarrow 0$ for $\alpha_{e}$ the well known result is obtained:

$\alpha_{c}(\omega)=\frac{4 \pi \omega}{n c} \operatorname{Im} \sum_{\mu} Z_{\mu}^{2} g_{\mu} \underset{\varepsilon \rightarrow 0}{=} \frac{2 \pi}{c} Z^{2}(\omega) D(\omega)$

where $D(\omega)$ is the density of states of the vibrational continuum and the index $\mu$ of $Z_{\mu}$ has been replaced by the continuously varying frequency $\omega$. This formula has been frequently used to describe impurity induced infrared absorption in defective crystals [10] and the absorption of amorphous materials [11]. The absorption coefficient reproduces the density of states multiplied by a generally frequency dependent oscillator strength which is in our terminology the square of the effective charge $Z(\omega)$ of the continuum.

The absorption of the discrete phonon $\alpha_{d}(\omega)$ is additively superimposed on $\alpha_{c}(\omega)$. Using equations 5 and 8 we get 
$\alpha_{d}(\omega)=\frac{4 \pi \omega}{n c} \frac{\operatorname{Re} F_{0}^{2} \omega \Gamma_{0}+\operatorname{Im} F_{0}^{2}\left(\Omega_{0}^{2}-\omega^{2}\right)}{\left(\Omega_{0}^{2}-\omega^{2}\right)^{2}+\left(\omega \Gamma_{0}\right)^{2}}$

with

$\operatorname{Re} F_{0}^{2}=\left(Z_{0}-\Lambda^{\prime}\right)^{2}-\Lambda^{\prime \prime 2}$

and

$\operatorname{Im} F_{0}^{2}=2 \Lambda^{\prime \prime}\left(\Lambda^{\prime}-Z_{0}\right)$.

Examining this equation, we see that when $\operatorname{Im} F_{0}^{2}=0$ we have the usual symmetric oscillator absorption line if we assume $\Delta$ and $A$ to be independent of frequency. The second term in the nominator yields an asymmetric additive contribution to the absorption profile due to the factor $\Omega_{0}^{2}-\omega^{2}$, which is only present if $F_{0}^{2}$ or equally $A$ has an imaginary part. This term gives the contribution of the interference between the discrete phonon and the continuum modes. We rewrite equation 12 and obtain for the total absorption coefficient

$\alpha(\omega)=\alpha_{c}(\omega)+\alpha_{d}^{(0)} \frac{1+W\left(\Omega_{0}^{2}-\omega^{2}\right) / \omega \Gamma_{0}}{1+\left(\Omega_{0}^{2}-\omega^{2}\right)^{2} /\left(\omega \Gamma_{0}\right)^{2}}$

with

$\alpha_{d}^{(0)}=\frac{4 \pi R e F_{0}^{2}}{n c \Gamma_{0}}=\frac{4 \pi\left\{\left(Z_{0}-\Lambda^{\prime}\right)^{2}-A^{\prime \prime 2}\right\}}{n c \Gamma_{0}}$

and

$W=\frac{\operatorname{Im} F_{0}^{2}}{\operatorname{Re} F_{0}^{2}}=\frac{2 \Lambda^{\prime \prime}\left(\Lambda^{\prime}-Z_{0}\right)}{\left(Z_{0}-\Lambda^{\prime}\right)^{2}-\Lambda^{\prime \prime 2}}$.

In this equation $W$ may be regarded as an asymmetry parameter, which vanishes for symmetric line shapes $\left(A^{\prime \prime}=0\right)$. The coefficient $\alpha_{d}^{(0)}$ is the absorption coefficient at the renormalized resonance frequency $\Omega_{0}$. It is connected with the integral absorption due to the discrete phonon by:

$\int \alpha_{d}(\omega) d \omega=\frac{\pi}{2} \Gamma_{0} \alpha_{d}^{(0)}$

if $\Gamma_{0} \ll \Omega_{0}$.

The parameter $\alpha_{d}^{(0)}$ may be modified by the phonon interaction, even if the absorption line is symmetric with $W=0$. If this situation applies, $\alpha_{d}^{(0)}$ is the peak absorption of the line measured above $\alpha_{c}$. Further we note that the depth of the antiresonance is limited by the thermodynamic requirement $\alpha=\alpha_{c}+\alpha_{d}>0$. This implies the upper boundary of the asymmetry parameter $|W|>2 \alpha_{c} / \alpha_{d}^{(0)}$.

In the case of a narrow resonance, $\Gamma_{0} \ll \Omega_{0}$, the expressions $\Omega_{0}^{2}-\omega^{2}$ and $\omega \Gamma_{0}$ in the above equations may be replaced by $\Omega_{0}^{2}-\omega^{2} \simeq$ $2 \Omega_{0}\left(\Omega_{0}-\omega\right)$ and $\omega \Gamma_{0} \simeq \Omega_{0} \Gamma_{0}$. Then it is easily shown that $\alpha(\omega)$ describes just a $F$ ano line shape function of the form:

$\alpha(\omega)=\alpha_{c}(\omega)-\sigma+\sigma \frac{(q+\varepsilon)^{2}}{\left(1+\varepsilon^{2}\right)}$

where $\varepsilon=\left(\omega-\Omega_{0}\right) /\left(\Gamma_{0} / 2\right)$ is the reduced energy variable and $\sigma=\frac{4 \pi}{n c} \frac{\left(\Lambda^{\prime \prime}\right)^{2}}{\Gamma_{0}}$ and $q=\left(Z_{0}-\Lambda^{\prime}\right) / \Lambda^{\prime \prime}$ are the Fano asymmetry parameters [6].

\section{Temperature dependence of the model parameters}

The model parameters $\alpha_{d}^{(0)}, W, \Omega_{0}$ and $\Gamma_{0}$, introduced in the last section, are generally frequency dependent quantities which are determined by the dynamics of PE. As we don't know the dynamical properties and particularly the coupling mechanism in the partially crystallized polymer, we will assume these parameters to be constant in the vicinity of the resonance frequency of the discrete phonon. This is obviously a very crude approximation, because the asymmetric absorption line extends at higher temperatures over about $20 \mathrm{~cm}^{-1}$ (see fig. 2), a frequency range where the background absorption $\alpha_{c}(\omega)$ shows a non-negligible variation. Therefore we had to specify for each temperature a frequency dependent absorption coefficient $\alpha_{c}(\omega)$ in order to obtain a reasonable coincidence between the experimental and theoretical absorption curves. This introduces a considerable uncertainty into the model parameters as derived from the experimental data. Figure 4 shows a typical fitted absorption curve with the hypothetical continuous absorption $\alpha_{c}(\omega)$. The renormalized resonance frequency $\Omega_{0}$ and the damping constant $\Gamma_{0}$ as functions of temperature determined by this fitting proce- 


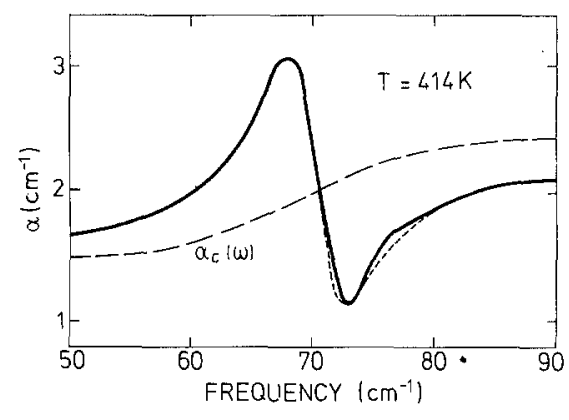

Fig. 4. Absorption coefficient $\alpha(\omega)$ at 414 K. Full line: experimental curve; broken line: fitted curve; $\alpha_{c}(\omega)$ : hypothetical continuum absorption

dure are given in figure 5 . For all temperatures we found $\Gamma_{0} \ll \Omega_{0}$. The damping constant is much smaller than the extension in frequency of the asymmetric absorption line. For large asymmetry, $W \gg 1, \Gamma_{0}$ is equal to the frequency difference between the peak absorption and the antiresonance minimum. Though in the case $\Gamma_{0} \ll \Omega_{0}$ the Fano formula equation 15 applies, we have evaluated $\alpha_{d}^{(0)}$ and $W$ after equation 13 because using these parameters the integral absorption determined by $\alpha_{d}^{(0)}$ and the asymmetry of the lineshape specified by $W$ are well separated. The renormalized resonance frequency $\Omega_{0}$ and the damping constant $\Gamma_{0}$ as functions of temperature determined by this fitting procedure are given in figure 5 and the results for $\alpha_{d}^{(0)}, W$ and $\alpha_{c}\left(\Omega_{0}\right)$ are shown in figure 6 . The coupling between the phonon continuum and the discrete phonon sets in at approximately $80 \mathrm{~K}$ and increases in strength with rising temperature. This is indicated by the continuous decrease of $\alpha_{d}^{(0)}$ and the frequency $\Omega_{0}$. Up to about $310 \mathrm{~K}$ the line shape

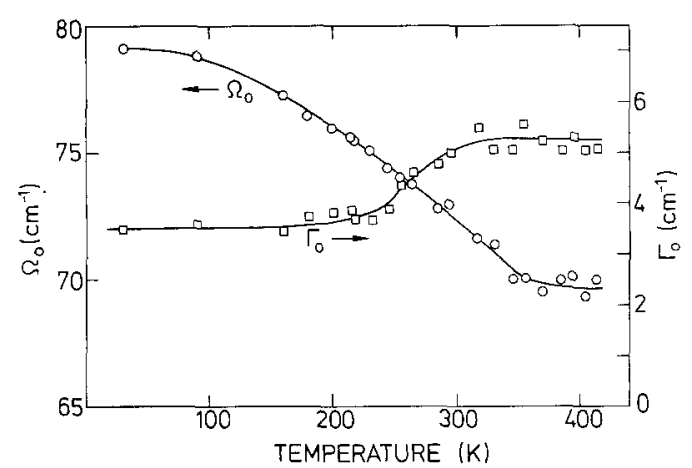

Fig. 5. Resonance frequency $\Omega_{0}$ and damping constant $\Gamma_{0}$ as functions of temperature is symmetric, thus $W=0$ and $A$ is real below this temperature. Also $\Delta^{\prime \prime}$ is very small up to $260 \mathrm{~K}$, which can be seen from the only slightly increasing damping constant $\Gamma_{0}$. Around $300 \mathrm{~K}$ the background absorption and the mode coupling change drastically. At $290 \mathrm{~K}$ the continuum absorption $\alpha_{c}\left(\Omega_{0}\right)$ rises within $10 \mathrm{~K}$ to twice it's lower temperature value. In the same temperature region the line width $\Gamma_{0}$ increases strongly and the line shape becomes asymmetric, thus $A$ is now complex yielding an asymmetry parameter $W>0$. When the temperature is further raised, $W$ increases strongly and the absorption strength $\alpha_{d}^{(0)}$ may be extrapolated to zero at about the melting temperature $T_{m}=430 \mathrm{~K}$ of crystalline PE.

\section{Discussion}

The coupling mechanism of the discrete $B_{1 u}$-phonon of crystalline PE to a continuum of infrared active modes introduces the question for the physical nature of this continuum. We discuss two conceivable mechanisms:

a) The PE crystallites with a mean edgelength of $300-500 \AA$ contain crystal defects of several species. Here the most important and at the same time simplest one is the so called "kink"-defect formed by two neighboured gauche positions of three successive $\mathrm{CH}_{2-}$ groups [12]. Because of the relaxation of the k-selection rule, such defects contribute to some extent to the continuous background absorption. This has been proved by investigating annealed material. Annealing lowers the number of defects in the crystallites and always reduces the background absorption. However, a strong influence of the kinks on the temperature behaviour of the line distortion is unlikely, since the concentration of kinks between room temperature and the melting point does not vary remarkably [13].

b) The continuum of vibrational modes of the amorphous parts of the material must also be taken into account. Again due to the lack of translational symmetry all modes of the amorphous portions are infrared active [11]. The coupling mechanism may be given by the transition of chains from the crystalline parts into the amorphous regions, leaving the crystallite surfaces in a distance of ca. $4 \AA$ corresponding to the $b$-axis length of the 


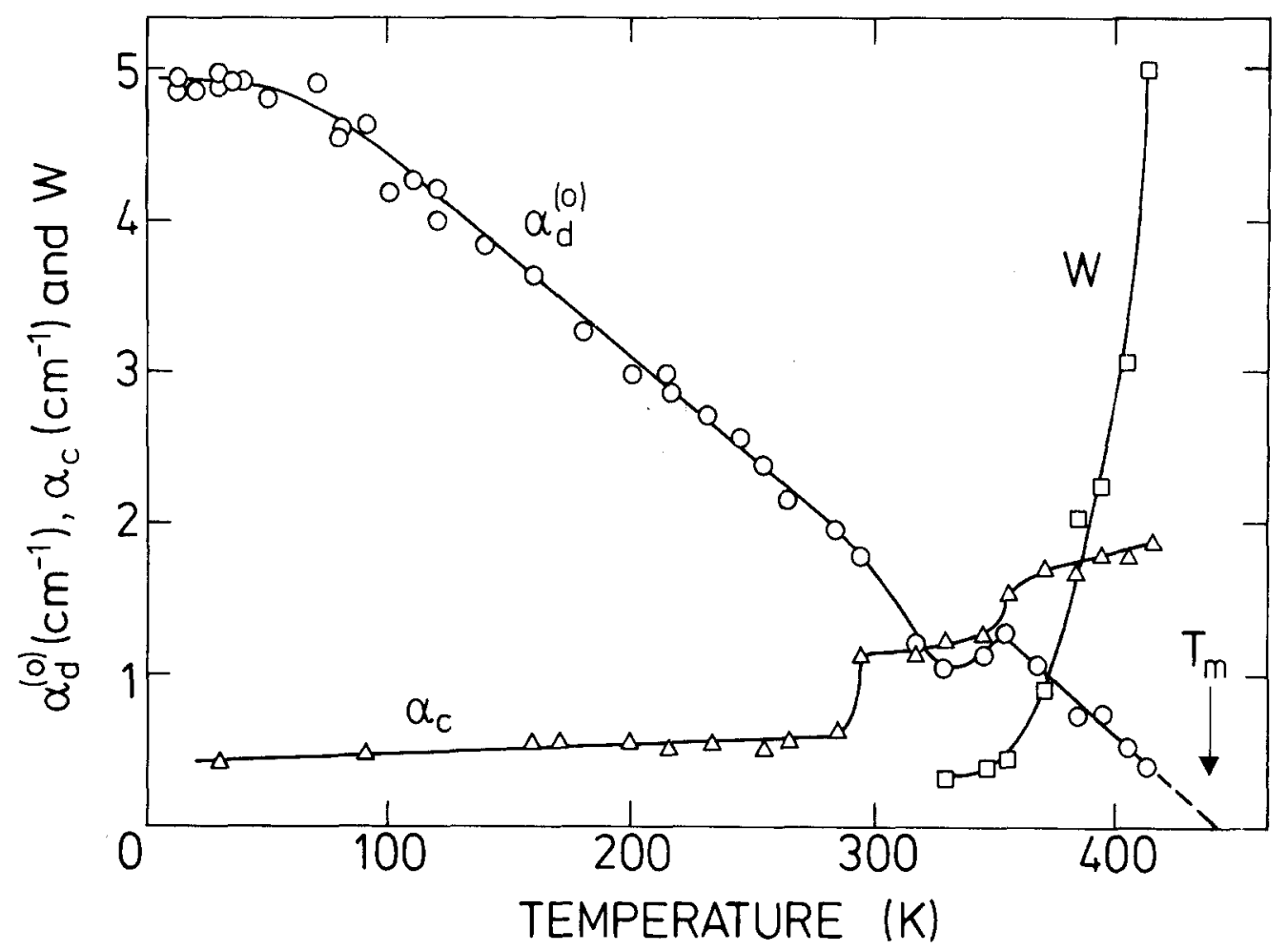

Fig. 6. Peak absorption $\alpha_{d}^{(0)}$, asymmetry parameter $W$ and continuum absorption $\alpha_{c}\left(\Omega_{0}\right)$ as functions of temperature. $T_{m}$ is the melting temperature of crystalline $\mathrm{PE}$

crystal. The similarity of the mean chain distance in the amorphous parts to the $b$-axis value can explain the observed fact, that the band intensity of the $73 \mathrm{~cm}^{-1}$ band is so much stronger than that of the $109 \mathrm{~cm}^{-1}$ (see fig. 1 and 2), an experimental observation having no stringent explanation up two now. From symmetry relations the two bands corresponding to two transversal vibrations in $b$-axisdirection $\left(73 \mathrm{~cm}^{-1}, B_{1 u}\right)$ and $a$-axis-direction $\left(109 \mathrm{~cm}^{-1}, B_{2 u}\right)$ should not vary drastically in their absorption intensity. Till now we have argued that the absorption strength of $73 \mathrm{~cm}^{-1}$ band and thus $Z_{0}$ is large at low temperatures and is diminished with rising temperature by the mode coupling. If we assume, that the effective charge of the discrete phonon of $73 \mathrm{~cm}^{-1} Z_{0}$ is equal or close to zero, than the effect would be still existent (see eq. 13), since $\Lambda^{\prime}$ would produce a "transfer" of absorption strength from the continuum to the discrete phonon. By the coupling via $b$-axis this would be only the case for the $73 \mathrm{~cm}^{-1}$ phonon, not for the $109 \mathrm{~cm}^{-1}$ mode.
In order to gain a complete understanding of the microscopic phonon processes in $\mathrm{PE}$ additional experiments are necessary. In particular materials with different crystallinity should be investigated. Furthermore other partially crystalline polymers are interesting, because it is very probable that phonon interactions of the type discussed here are not restricted to $\mathrm{PE}$.

\section{Summary}

The decrease of the absorption strength of $73 \mathrm{~cm}^{-1}$ line in polyethylene and the strong antiresonance occuring above $310 \mathrm{~K}$ at the high frequency wing of the line are explained by assuming a Fanto type coupling between the $73 \mathrm{~cm}^{-1}$ phonon and a continuum of infrared active vibrational modes. The parameters determining the line shape are deduced from experimental absorption curves and conceivable coupling mechanisms are discussed.

\section{Zusammenfassung}

Die Abnahme der Absorption der $73 \mathrm{~cm}^{-1}$-Bande in Polyäthylen und die starke Antiresonanz, die oberhalb $310 \mathrm{~K}$ auf der hochfrequenten Seite der Bande einsetzt, werden erklärt durch Annahme einer 
Fano-Kopplung zwischen dem $73 \mathrm{~cm}^{-1}$-Phonon und einem Kontinuum von infrarot-aktiven Schwingungsmoden, Die Parameter, die die Linienform bestimmen, werden aus den experimentellen Absorptionskurven abgeleitet und Kopplungsmechanismen diskutiert.

\section{Acknowledgement}

One of us (W. F. X. Frank) is indebt to the Deutsche Forschungsgemeinschaft for financial support (Project Fr 442/5 and $7 \mathrm{~b}$ ).

\section{References} (1964).

1) Bertie, I. E., E. Whalley, J. Chem. Phys. 41, 575

2) Dean, G. D., B. H. Martin, Chem. Phys. Lett. 1,415 (1967).

3) Tasumi, M., T. Sbimanoucbi, J. Chem. Phys. 43, 1245 (1965). (1967).

4) Tasumi, M., S. Krimm, J. Chem. Phys. 46, 755

5) Frank, WV., H. Schmidt, W. Wulf, J. Polymer Science: Polymer Symposium 61, 317 (1977).

6) Fano, U., Phys. Rev. 124, 1866 (1961).

7) Scott, J. F., Rev. Mod. Phys. 46, 83 (1974).

8) Balkanski, M., K. P. Jain, R. Beserman, $M$. Jonanne, Phys. Rev. B12, 4328 (1975).
9) Barker, A. S., J. J. Hopfield, Phys. Rev. 135, A 1732 (1964).

10) Klein, M.V., Physics of Color Centers, Chap. 7, Ed. W. Beall Fowler, Academic Press (New York 1968).

11) Prettl, W., N. Sbevcbik, M. Cardona, phys. stat. sol. (b) 59, 241 (1973).

12) Pecbold, W., S. Blasenbrey, Kolloid-Z. u. Z. Polymere 216/217, 235 (1967).

13) Scherr, S., Kolloid-Z. u. Z. Polymere 252, 871 (1974).

Authors' addresses:

W. Prettl

Institut für Angewandte Physik der

Universität Regensburg

Universitätsstr. 31

D-8400 Regensburg

W. F.X. Frank

Abteilung Experimentelle Physik III der

Universität Ulm

Oberer Eselsberg

D-7900 Ulm 